\title{
Diagnostic yield of rare skeletal dysplasia conditions in the radiogenomics era
}

\author{
Ataf H. Sabir ${ }^{1,2^{*}}$, Elizabeth Morley ${ }^{3}$, Jameela Sheikh², Alistair D. Calder ${ }^{4}$, Ana Beleza-Meireles ${ }^{5}$, \\ Moira S. Cheung ${ }^{6}$, Alessandra Cocca ${ }^{6}$, Mattias Jansson', Suzanne Lillis ${ }^{7}$, Yogen Patel $^{8}$, Shu Yau', \\ Christine M. Hall ${ }^{9,10}$, Amaka C. Offiah ${ }^{11}$ and Melita Irving ${ }^{1,12}$
}

\begin{abstract}
Background: Skeletal dysplasia (SD) conditions are rare genetic diseases of the skeleton, encompassing a heterogeneous group of over 400 disorders, and represent approximately $5 \%$ of all congenital anomalies. Developments in genetic and treatment technologies are leading to unparalleled therapeutic advances; thus, it is more important than ever to molecularly confirm SD conditions. Data on 'rates-of-molecular yields' in SD conditions, through exome sequencing approaches, is limited. Figures of 39\% and 52.5\% have been reported in the USA $(n=54)$ and South Korea $(n=185)$ respectively.
\end{abstract}

Methods: We discuss a single-centre (in the UK) experience of whole-exome sequencing (WES) in a cohort of 15 paediatric patients (aged 5 months to 12 years) with SD disorders previously molecularly unconfirmed. Our cohort included patients with known clinical diagnoses and undiagnosed skeletal syndromes. Extensive phenotyping and expert radiological review by a panel of international SD radiology experts, coupled with a complex bioinformatics pipeline, allowed for both gene-targeted and gene-agnostic approaches.

Results: Significant variants leading to a likely or confirmed diagnosis were identified in $53.3 \%(n=8 / 15)$ of patients; $46.7 \%(n=7 / 15)$ having a definite molecular diagnosis and $6.7 \%(n=1 / 15)$ having a likely molecular diagnosis. We discuss this in the context of a rare disease in general and specifically SD presentations. Of patients with known diagnoses pre-WES $(n=10)$, molecular confirmation occurred in $7 / 10$ cases, as opposed to $1 / 5$ where a diagnosis was unknown pre-test. Thus, diagnostic return is greatest where the diagnosis is known pre-test. For WGS (whole genome sequencing, the next iteration of WES), careful case selection (ideally of known diagnoses pre-test) will yield highest returns.

Conclusions: Our results highlight the cost-effective use of WES-targeted bioinformatic analysis as a diagnostic tool for SD, particularly patients with presumed SD, where detailed phenotyping is essential. Thorough co-ordinated clinical evaluation between clinical, radiological, and molecular teams is essential for improved yield and clinical care. WES (and WGS) yields will increase with time, allowing faster diagnoses, avoiding needless investigations, ensuring individualised patient care and patient reassurance. Further diagnoses will lead to increased information on natural history/mechanistic details, and likely increased therapies and clinical trials.

Keywords: Mendelian, Molecular genetic test, Monogenic, Next-generation sequencing, Skeletal dysplasia, Exome sequencing, Genome sequencing, Yield

\footnotetext{
*Correspondence: ataf.sabir2@nhs.net

${ }^{1}$ Department of Clinical Genetics, Guy's and St Thomas' NHS Foundation

Trust, London, UK

Full list of author information is available at the end of the article
} 


\section{Background}

Rare diseases by definition affect less than 1 in 2,000 (in Europe) or 1 in 1,500 (in the USA). Approximately $80 \%$ of the 7300 rare diseases have a known genetic cause, and whole-exome sequencing (WES) is commonly the final diagnostic step in such cases with diagnostic yields averaging around 31\% (24-68\%) [1-3].

The broad range in WES diagnostic yields (see Table 1) reflects varying cohorts and clinical indications for testing (i.e., diagnostic rates are higher in selected cohorts with conspicuous phenotypes). Yield is highly influenced by recruitment type (e.g., single proband versus trio), bioinformatic pipelines, study year, consanguinity, case complexity and degree of prior genetic evaluation. Yields tend to increase with study year (due to increasing novel genes, conditions, and technological advances) and in cohorts with a high degree of consanguinity [2]. Conversely yields are lower in cohorts with highly complex cases necessitating challenging interpretation, e.g. cases where two or three independent genetic diagnoses lead to an aggregate phenotype. Yield is also markedly affected by the extent of prior genetic and metabolic evaluation; thus, WES usage earlier in the diagnostic assessment can lead to higher yields.

\section{Skeletal dysplasia cohorts}

The majority of rare diseases (50-75\%) affect children [10]. In paediatric unsolved rare disease cohorts, the diagnostic yield from exome sequencing is around $40 \%$ [11]. An important subset of rare paediatric disease is skeletal dysplasia (SD). SD conditions are rare disorders of the skeleton, encompassing a genetically heterogeneous group of over 400 distinct disorders. They represent approximately $5 \%$ of all congenital anomalies and are a significant contributory cause of children with severe short stature [12].

\section{Yields in SD}

Data on WES yields in SD cohorts are limited. In a large study of over 3000 rare diseases WES cases in a single US centre, Retterer et al., (2015) performed a subgroup analysis of 54 SD cases reporting a diagnostic yield of 39\% $(\mathrm{n}=54)$ [13]. Recruitment was predominantly trio based ( $>75 \%$ were trios, $7 \%$ were duos) though the patients were at different points of their diagnostic evaluation. The average patient age was $11-/+13$ years.

Bae et al. (2015) studied a large cohort of 185 patients with SD across multiple centres in South Korea, who had panel-based targeted exome sequencing (TES) (255 gene panel); 25 had a prior confirmed clinical and molecular diagnosis. Thus, TES was used to reconfirm molecular findings, 64 had an unknown clinical diagnosis, and 96 patients had an assured clinical diagnosis [14]. A molecular diagnosis was 'confirmed' or 'highly pathogenic' in $20 \%(n=13 / 64)$ of patients with an unknown clinical diagnosis and $71 \%(n=71 / 96)$ of patients with an assured clinical diagnosis. In this report, we explore the utility of WES in a cohort solely composed of SD presentations and consider the diagnostic yield in sub-cohorts, i.e., those with an unknown diagnosis and those with an assured diagnosis (either single gene or heterogeneous). Although the number of patients in our study is small $(n=15)$, to the best of our knowledge, there are no previous reports of a UK/European cohort of patients with SD reported.

\section{Methods}

\section{Subjects and clinical diagnosis}

All patients were study participants at the Guy's and St Thomas' Hospital clinical site. Our cohort spans the period; September 2014-2018. All patients within our cohort were paediatric ( 5 months to 12 years, mean age 5.5 years).

15 patients with presumed SD conditions based on clinical and radiographic findings, were selected from the specialist SD multidisciplinary clinic. The majority

Table 1 Diagnostic yield from NGS strategies in different rare disease cohorts in key publications

\begin{tabular}{llllll}
\hline Published year & Journal & Strategy & Sequenced & Diagnostic yield (\%) & Author \\
\hline 2013 & NEJM & WES & 250 & 24.8 & Yang et al. [4] \\
2014 & AJHG & WES & 264 & 55.3 & Beaulieu et al. [5] \\
2015 & Nat Genet & WGS* & 156 & 21.2 & Taylor et al. [6] \\
2017 & JAMA Pediatr & WES & 44 & 52.3 & Tan et al. [7] \\
2017 & JAMA Pediatr & WES & 278 & 36.7 & Meng et al. [8] \\
2017 & JAMA Pediatr & Critical trio WES & 63 & 50.8 & Meng et al. [8] \\
2018 & NPJ Genome Med & WGS* & 42 & 42.9 & Farnaes et al. [9] \\
2019 & Sci Rep & WGS* & 10 & 62.5 & Liu et al. [3] \\
\hline
\end{tabular}

WES whole-exome sequencing; WGS whole-genome sequencing 
of participants were referred to the service from external facilities. Informed consent was obtained for all patients, and thereafter genomic DNA was extracted from the proband and where possible their parents. Many participants were at different stages of their evaluation.

Patients were recruited as trios (proband and both parents) unless otherwise stated.

The 15 patients were divided into three categories based on the certainty of clinical diagnosis and the status of the prospective genotype. Patients with an assured clinical diagnosis of an SD, where one or only a few genotypes are known to be responsible (e.g., achondroplasia) were excluded as they are not appropriate for WES unless single gene testing was not readily available. Patients were then assigned one of three categories.

- Known condition-known gene (Category K-K)

- Unknown condition-unknown gene (Category U-U)

- Known condition/group of conditions-the possibility of multiple genes (Category $\mathrm{K}-\mathrm{U}$ )

\section{Exome sequencing and variant prioritisation}

WES capture was performed using Agilent SureSelectXT Human All Exon V5 baited with clinically relevant genes followed by sequencing on an Illumina HiSeq 2500 (though three of the later cases were sequenced on an Illumina NextSEQ 550). Raw sequence data were aligned using Novoalign, and variants called with Samtools. Coverage of coding exons $(+/-5$ base pairs) was to a minimum depth of $\times 20$.

\section{Variant analysis}

Qiagen Ingenuity Variant Analysis was used to aid the assessment of variant pathogenicity after applying a virtual panel of 222 SD genes (see Appendix 1) in combination with multidisciplinary clinical interpretation. Qiagen analysis uses (CGI 54 Genomes, SIFT, Exome Variant Server (EVS), Allele Frequency Community, JASPAR, Ingenuity Knowledge Base, Vista Enhancer, BSIFT, TCGA, PolyPhen-2, 1000 Genome Frequency, Clinvar, COSMIC, ExAC, HGMD, PhyloP, DbSNP, TargetScan), and Alamut for splice site analysis (SpliceSiteFinder-like, MaxEntScan, NNSplice, GeneSplicer).

Sequencing data were analysed in three stages. In stage one, clinicians proposed primary genes or small genepanels for analysis through the WES platform that were likely to harbour the causative variant. This reduced the volume and cost of variants to analyse. If no significant variants were identified, then stage two involved applying and interpreting the 222 SD virtual gene-panel (see Appendix 1). If no appropriate variant was identified in stage two, then stage three involved a human phenotype ontology (HPO) driven whole-exome wide-search. HPO terms were derived by clinical geneticist expert review along with medical record evaluation and blinded radiographic review by three independent $\mathrm{SD}$ expert radiologists $(\mathrm{CH} / \mathrm{AO} / \mathrm{AC})$. The phenotypic terms and differential diagnosis provided were critical for analysis.

\section{Molecular diagnosis using determined variants}

Molecular diagnosis was made by correlating clinical and radiographic findings with candidate sequence variants through WES. The status of the molecular diagnosis was determined as either known or unknown.

\section{Results}

Our cohort of 15 patients comprised: five males and ten females, with ages ranging from 5 months to 12 years with an average age of 5.5 years old. Significant variants were identified in $53.3 \%(n=8 / 15)$ of patients; $46.7 \%$ $(n=7 / 15)$ having a definite molecular diagnosis and 6.7\% $(n=1 / 15)$ having a likely molecular diagnosis. One case (patient 15) after negative WES testing was concluded to have acquired SD aetiology. Thus, $60 \%(n=9 / 15)$ cases had a confirmed or likely confirmed diagnosis (yield: $60 \%)$.

Of unknown clinical diagnosis $(n=5 / 15)$, WES led to molecular confirmation (likely or highly likely) in $20 \%$ $(n=1 / 5)$.

\section{Discussion}

SD diagnoses are challenging for several reasons. Firstly, similar phenotypes are heterogeneous; one gene can cause several conditions. Secondly, the aetiology of multiple SD conditions is unknown or their phenotype not well established. Thirdly, the experience of SD conditions by individual clinicians is limited, and lastly, the characteristic features of many SD conditions only manifest at certain periods. For example, stippled epiphyses (characteristic of chondrodysplasia punctata) are only present in the neonatal or infantile period; thus, diagnoses are difficult without timely investigation. Similarly, many characteristic features disappear after skeletal maturity or only present when young. Multidisciplinary approaches, involving geneticists, radiologists, orthopaedic, growth specialists and therapists (occupational, physiotherapy, psychological), are often necessary for diagnosis and management of SD conditions.

Using a single-gene testing strategy is often unhelpful for the reasons stated, and increasingly WES or WGS options are deployed. In some countries specific panels are used, in place of or prior to WES/WGS, due to lower costs, accessibility and relatively large numbers of genes specific to the phenotype. Infact many WES or WGS 
approaches begin with panel-based analysis before more agnostic or wider analysis. Little is reported regarding the diagnostic yield of WES in SD conditions, as often such cases are pooled in general rare disease cohorts. Where rates and analysis have been reported, little is discussed around the prior categorisation of cases (e.g., whether the diagnosis was known).

Despite these challenges, yields for SD conditions are relatively high compared to other rare disease categories. In fact, for fetal anomalies, the highest yields $(\sim 80 \%)$ were obtained in the SD category in the UK fetal exome PAGE (prenatal assessment of genomes and exomes) study [15]. However, the yield is less spectacular postnatally. Retterer et al. report a yield of 39\% in SD patients $(\mathrm{n}=54)$ using WES in a combined paediatric and adult cohort [13]. Bae et al., reported a likely or confirmed molecular diagnosis in 52.5\% $(n=84 / 160)$ through TES, though the patient ages are not reported [14].

To diagnose SD conditions, radiographic imaging in early childhood is key; thus, our cohort of only paediatric patients consisted of suitably appropriate clinicalradiographic data for further genetic investigation. None of the patients had molecular confirmation of their condition before WES testing. Upon WES testing, a threestage approach to variant analysis (as outlined in the methods) was undertaken. This allowed molecular scientists to focus their search, identifying pathogenic variants efficiently and saved time spent analysing less likely variants.

Significant variants leading to a likely or confirmed diagnosis were identified in $53.3 \%(n=8 / 15)$ of patients; $46.7 \%(n=7 / 15)$ having a definite molecular diagnosis and $6.7 \%(\mathrm{n}=1 / 15)$ having a likely molecular diagnosis. In comparison, Bae et al. reported a likely or confirmed molecular diagnosis in 52.5\% $(n=84 / 160)$ of similar patients (excluding those patients who had molecular confirmation prior to WES testing) [14]. This means that our yield was comparable to Bae et al., and was marginally higher. If we include patient 15 as a confirmed diagnosis, who after negative WES testing was concluded to have an acquired SD aetiology, then our yield reached $60 \%(n=9 / 15)$.

The possible reasons for a slightly higher yield as compared to Bae et al. includes increased gene discovery linked to SD conditions since Bae et al. was published (2016), improved bioinformatic pipelines, technological advances in next-generation sequencing machinery (e.g. improved coverage and read depth) and triple radiological review within our analysis (all three reviewers being expert contributors to the International Skeletal Dysplasia Society) [14]. We note that radiological input was key with patients 2 and 4 . For patient 2, the radiology review led to a change in the pre-test working diagnosis from spondyloepiphyseal dysplasia congenita (SEDC) to campomelic dysplasia (CD), enabling a better genotypephenotype match, thus securing the diagnosis. Likewise, the radiological diagnosis with patient 4 identified the correct spectrum of disorders so that when a COL11A2 (collagen type XI alpha-2 chain) variant was found, the appropriate final diagnosis was more easily identified.

Of patients with a known diagnosis pre-WES $(n=10)$, WES led to a confirmed molecular diagnosis in $7 / 10$ cases (rising to $8 / 10$ if we include patient 8 as another confirmed diagnosis, though of an acquired cause). This highlights the significant return when there is a known diagnosis pre-test.

Of patients with unknown clinical diagnoses $(n=5 / 15)$, WES led to molecular confirmation (likely or highly likely) in $20 \%(n=1 / 5)$ of cases which is the same detection rate as Bae et al. [14] This was expected considering low pre-test diagnostic SD hypotheses.

WES yields for non-SD conditions (25-51\%, see Table 1) are generally lower than for SD disorders (this study; up to $60 \%$ and Bae et al. 52.5\%) [14]. This is likely due to the rich combination of radiology and clinical features, and synergism between multiple experts, enhancing phenotypic-driven bioinformatics analysis.

We further discuss selected patients to extrapolate key learning points and have grouped the patients into themes.

\section{Theme 1: known clinical diagnosis with clear single-gene cause, but testing unavailable}

Patient 1 had a clinical diagnosis of spondyloepiphyseal dysplasia tarda (SEDT; OMIM \#313,400). SEDT is caused by heterozygous TRAPPC2 (tracking protein particle complex subunit 2) variants. Several males across multiple generations had a similar diagnosis but no known genetic testing. At the time of diagnosis in patient 1 (2014), TRAPPC2 testing was not readily available in the UK (except on a research basis) [16]. Thus, WES was performed, with primary analysis directed towards TRAPPC2. A pathogenic TRAPPC2 variant was identified (see Table 2). With many laboratories moving to WES and WGS, the availability of many single-gene tests is decreasing; thus, WES approaches with targeted analysis are becoming ubiquitous. It is therefore crucial that clinicians make a pre-test diagnosis to enable targeted analysis and prevent the generation of unwanted and unrelated variants.

\section{Theme 2: known clinical diagnosis (heterogeneous condition), multiple potential genetic causes}

Patient 4 had macrocephaly, a flat facial profile, nasal bridge depression, small nose, micrognathia, a small bell-shaped thorax and short long-bones with widened 


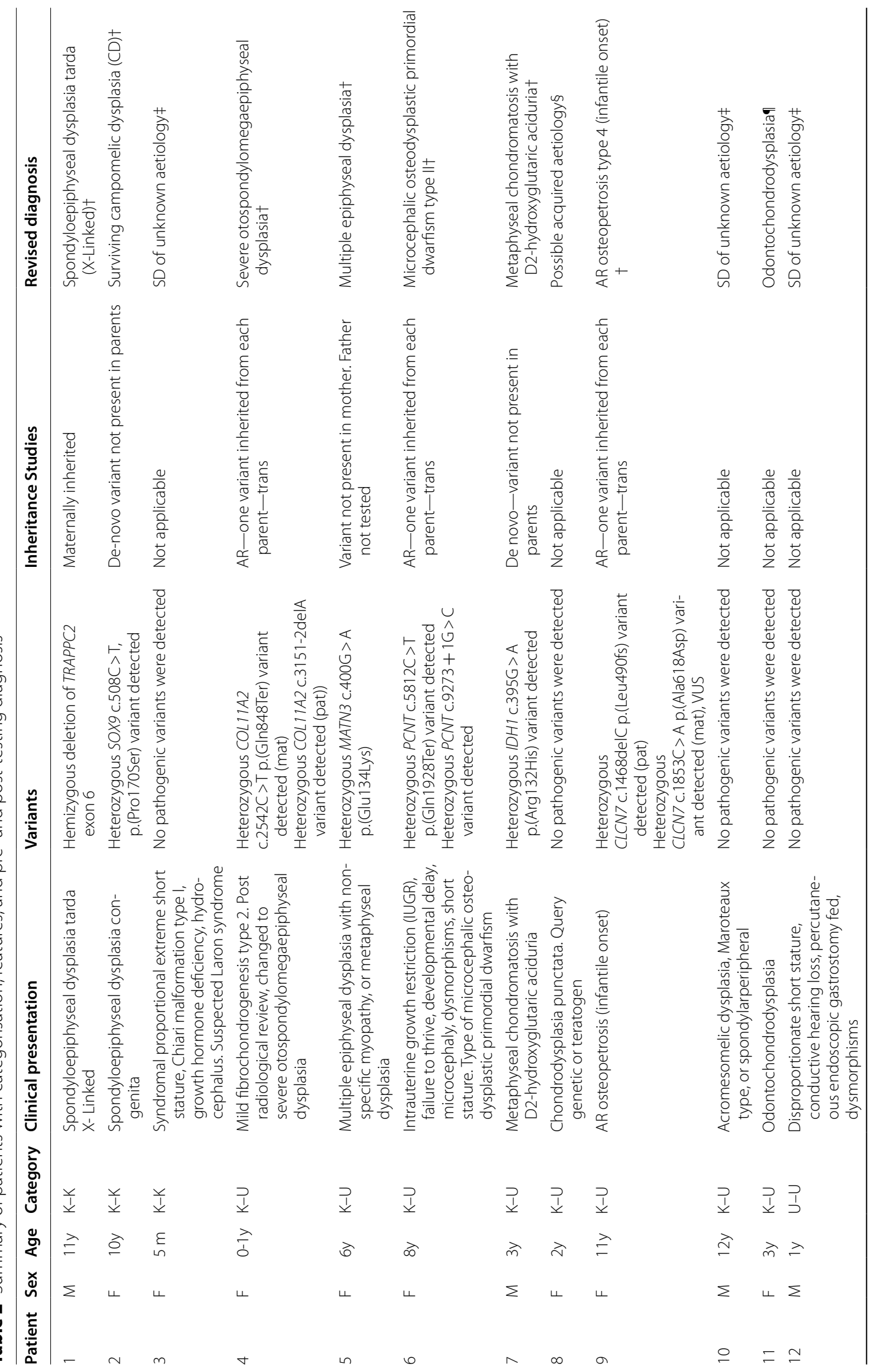


Sabin et al. BMC Med Genomics <wide> (2021) 14:148

Page 6 of 14

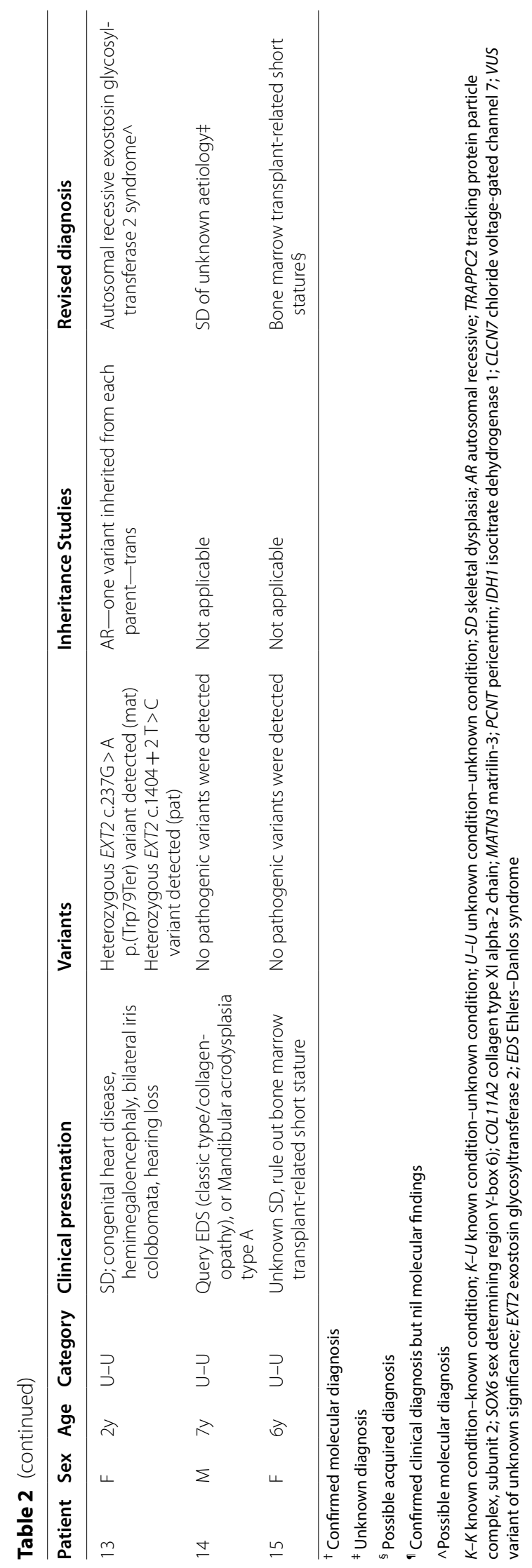


metaphyses. The original diagnosis was 'mild' fibrochondrogenesis type 2 (FBCG2; OMIM \#614524), which is caused by COL11A2 variants and can be lethal. The differential included fibrochondrogenesis type 1 (OMIM \#228520) caused by COL11A1 as well as Stickler syndrome (OMIM \#108300) hence multiple genes could be potentially causative; thus, WES was an appropriate strategy. The radiological review noted vertebral coronal and sagittal clefts and with increasing age (see Fig. 1); enlarged epiphyses and clinically hearing loss became manifest. This led to an alteration of the diagnosis to otospondylomegaepiphyseal dysplasia (OSMED; OMIM \#215150), an allelic disorder to FBCG2. WES confirmed compound heterozygous COL11A2 variants which cause a spectrum of disorders from mild deafness to OSMED to potentially lethal FBCG2. This case illustrated radiological (e.g. mega-epiphyses) and clinical clues (hearing loss) that may only become apparent with time. It also highlights the need for clinicians to be careful of using 'old' diagnostics labels as milder forms of previously 'lethal' conditions or what we considered 'extremely' severe phenotypes are emerging. In such instances, umbrella terms like COL11A2-spectrum disorder as a pre-molecular diagnosis may be more apt rather than FBCG2.

Patient 5, a six-year-old male of normal stature who presented with leg pain, genu valgum, pes planus, muscle weakness and decreased hip mobility. His clinical picture and radiology suggested possible multiple epiphyseal dysplasia (MED; OMIM \#132400) with nonspecific myopathy. Creatinine kinase was normal, and electromyography suggested myopathic disorder, muscle biopsy showed mild type 2 fibre atrophy and array Comparative Genomic Hybridisation (aCGH) was normal. MED is characterised by multiple long and or short bone epiphyseal abnormalities and has autosomal dominant (ADMED; OMIM \#132400) and autosomal recessive (ARMED; OMIM \#226900) forms [17, 18]. ADMED can be due to; COMP (cartilage oligomeric matrix protein), COL9A1-COL9A3 (collagen type IX alpha 1-3 chain) or MATN3 (matrilin 3) and presents in childhood with joint pain, exercise-induced fatigue, restricted mobility, short stature, and early-onset osteoarthritis [18]. ARMED is caused by SLC26A2 (solute carrier family 26, member A2) formerly known as DTDST (diastrophic dysplasia sulfate transporter) and presents with joint pain and often mild short stature. SLC6A2 is responsible for three allelic skeletal dysplasias; (in increasing severity) diastrophic dysplasia, atelosteogenesis 2 and achondrogenesis type 1B [17]. Further key ARMED differentials include; mild mucolipidosis III (OMIM \#252605) and mucopolysaccharidosis VI (OMIM \#253000), both recessive storage disorders discriminated from ARMED by coarse dysmorphic facies, intellectual disability, visceral involvement, marked spondylar disease and shorter stature [1].

Radiology showed delayed epiphyseal (long-bone) ossification and small and irregular epiphyses (especially in the knees and hips) (see Fig. 2). Due to MED heterogeneity, WES was an appropriate strategy for molecular diagnosis and identified a pathogenic MATN3 variant confirming MED Type 5 (OMIM \#607078).

Patient 7 presented aged 3 years with an undefined SD, multiple falls, complex congenital heart disease (CCHD), relative macrocephaly and mild developmental delay. Skeletal survey at 10 months highlighted irregular metaphyses. He had leg-length discrepancy, right leg bowing, mild joint hypermobility and hypotonia. Further radiology (Fig. 3a, b) revealed worsening metaphyseal dysplasia (expansion, cup-shaped irregularity) with radiolucent non-ossified cartilage affecting major joints. At 6.5 years, hand radiology showed severe widespread bilateral multiple enchondromas (see Fig. 3c), that were also present in the lower limbs (see Fig. 3a). The diagnosis was felt to be an enchondromatosis-like condition and upon radiological review; metaphyseal chondromatosis with D-2-hydroxyglutaric aciduria (MC-D2HGA; OMIM \#614875) was suspected. Though MC-D2HGA was highly suspected, other genetic disorders with very similar phenotypes remained thus WES was an appropriate strategy.

Enchondromatosis (EC) is a rare heterogeneous condition with multiple enchondromas (benign hyaline cartilage forming tumours in the metaphysis) [19]. The commonest two subtypes are Ollier disease (OMIM \#166000) and then Maffucci syndrome (OMIM \#614569) [19]. MC-D2HGA is another rarer subtype with only 11 reported cases (four of which are due to somatic mosaicism of IDH1 (isocitrate dehydrogenase 1), the remainder lacking molecular confirmation) (Fig. 4) [20].

WES testing identified a de novo heterozygous $I D H 1$ c.395G > A p.(Arg132His) germline variant (approximately indicative allele frequency of 50/50, wild type: a variant on Sanger). Urinary organic acid testing showed marked isolated increase in 2-hydroxyglutarate, confirming MC-D2HGA (this result came after WES was initiated but prior to the WEST results). MC-D2HGA is associated with macrocephaly, developmental delay, hypotonia, significant metaphyseal dysplasia, enchondromatosis, dysmorphia and CCHD. This is the first reported MC-D2HGA case caused by germline IDH1 changes (with read depth over the IDH1 variant position of $>\times 250$ depth) though the author is aware of one unreported MC-D2HGA case caused by germline changes (Zankl A. [Presentation] 6th Nordic Workshop on Skeletal Dysplasia, Karolinska Institute, Sweden. 5th March 2020.) Had a WES approach not been used, it would have been difficult to reach this diagnosis. 


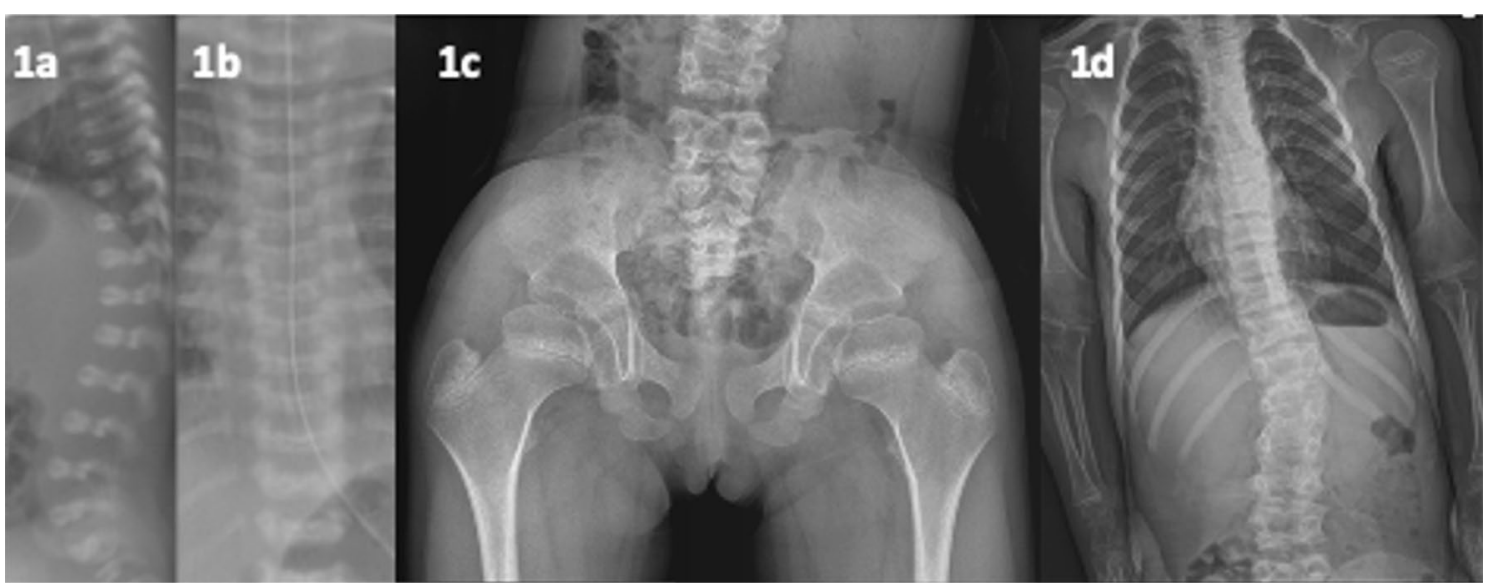

Fig. 1 (patient 4, OSMED). a and $\mathbf{b}$ Age day 3; sagittal spinal clefts, coronal spinal clefts. $\mathbf{c}$ and $\mathbf{d}$ age 6 years; mega-epiphyses of the femoral head, wide metaphyses, C-shaped left convexity of the spine, marked anterior wedging of T11, T12 and L1

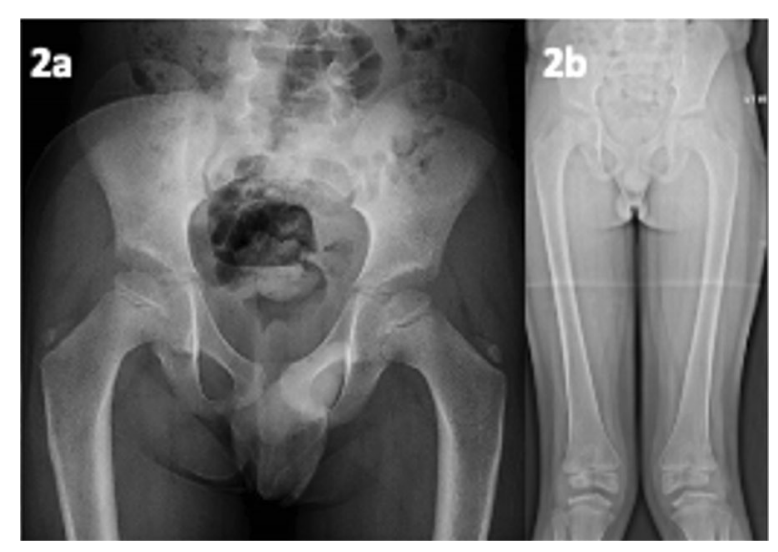

Fig. 2 (patient 5, MED). a Age 7 years; bilateral femoral head epiphyseal dysplasia, medial aspects fragmented. b Age 11 years; genu valgum and underdeveloped distal medial femoral epiphyses

Patient 9 presented with osteopetrosis, a heterogeneous condition, classically divided into; fatal infantile malignant form (OMIM \#259700/611490/259720), Albers-Schonberg disease (OMIM \#166600/259700) and a milder adult form. She presented with facial nerve palsy, and subsequent radiography noted Rickettsial-like bony ends (see Fig. 5). Foraminal impingement led to hearing loss and visual problems and a working diagnosis of infantile-onset osteopetrosis was established, suitable for WES. The proband had compound heterozygous variants in CLCN7 (chloride voltagegated channel 7); a pathogenic variant inherited from her unaffected father and a VUS (variant of unknown significance) from her unaffected mother, though her maternal grandmother was affected (late-onset osteopetrosis) and had the same VUS. It later transpired that the mother actually had mild radiographic and clinical features demonstrating segregation and striking intrafamilial variable expression of osteopetrosis type 4 (OMIM \#611490). If PP4 (protein phosphate-4) or PP1 (protein phosphate-1) panels were applied, the VUS would be upgraded to likely pathogenic. The paternal variant alone did not manifestly cause disease in the father but was felt to negatively modify the severity of the condition in the presence of the maternal variant.

\section{Theme 3: known diagnosis, no known gene}

In 2015, Patient 11, aged 3,presented with severe short stature, delayed motor milestones, a high-pitched voice, dysmorphism, ligamentous laxity, instability of the cervical spine, severe scoliosis (see Fig. 6a, b), discoloured/ weak dentition (see Fig. 6c), and no fractures. The working diagnosis after radiological review was a rare form of 'spondylometaphyseal dysplasia with dentinogenesis imperfecta, odontochondrodysplasia (OMIM \#184260). At the time, this condition had no known gene cause. Thus, an agnostic approach via WES was appropriate for new gene discovery. No pathogenic variants were found. Some years later, a causative gene was discovered for the condition, TRIP11(thyroid receptor-interacting protein 11) though despite re-analysis, a molecular cause was not confirmed in patient 11 . Other genetic causes of odontochondrodysplasia are still sought.

\section{Theme 4: unknown SD diagnosis}

Patient 13 presented with SD (short stature, macrocephaly, and scoliosis) with non-skeletal syndromal features (congenital heart disease and hearing loss). CHD7 (chromodomain helicase DNA binding protein 7) gene testing 


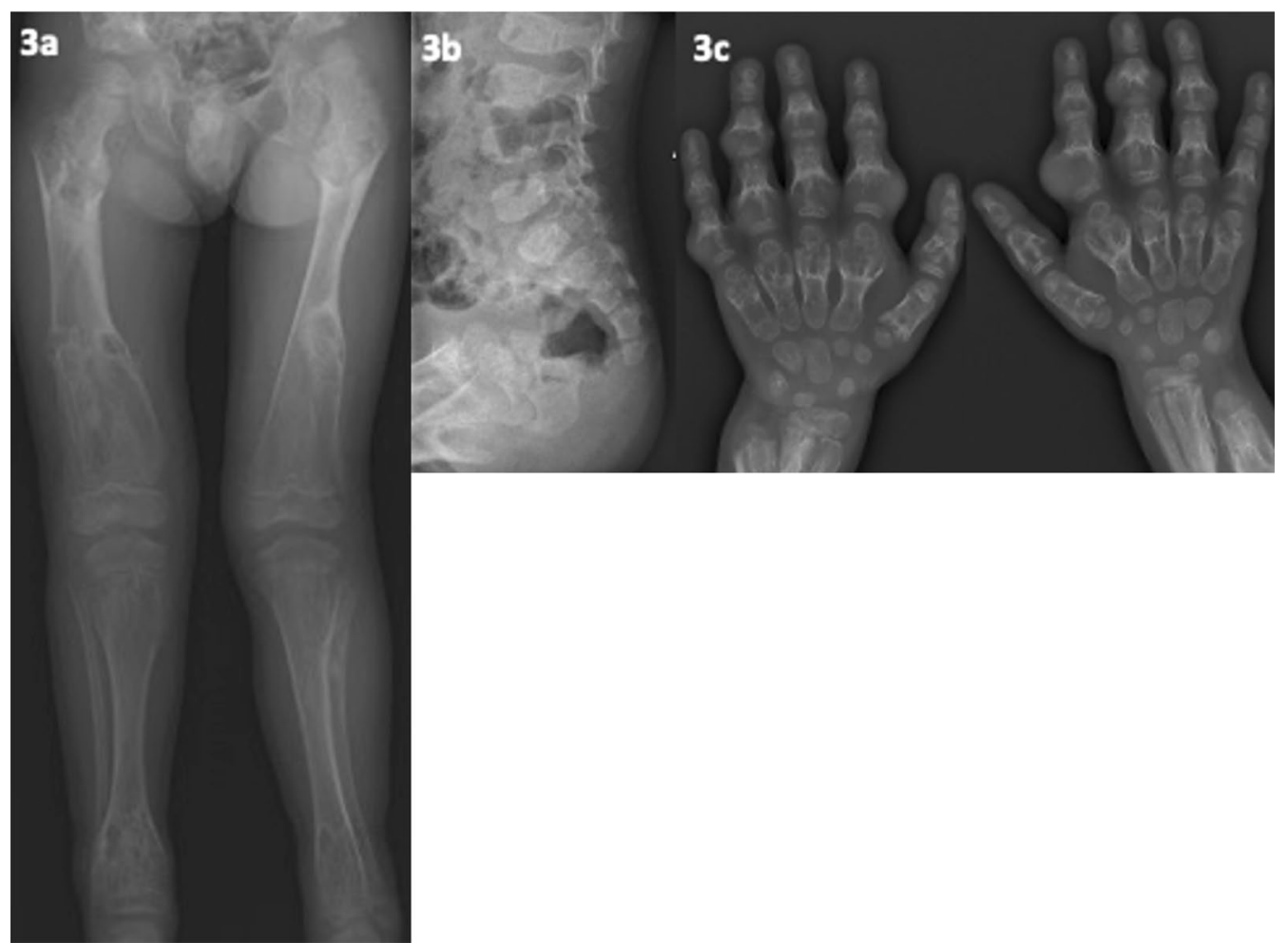

Fig. 3 (patient 7, metaphyseal chondrodysplasia-D2-HGA type). a Age 7 years; left leg is longer than right, marked left-sided genu valgum. Multiple expansile lucent metaphyseal lesions involving bilateral femora and tibia. b Age 2.5 years; expansion and irregularities of vertebral bodies. $\mathbf{c}$ Age 7 years; extensive enchondromatosis of both hands and wrists with associated soft tissue swelling

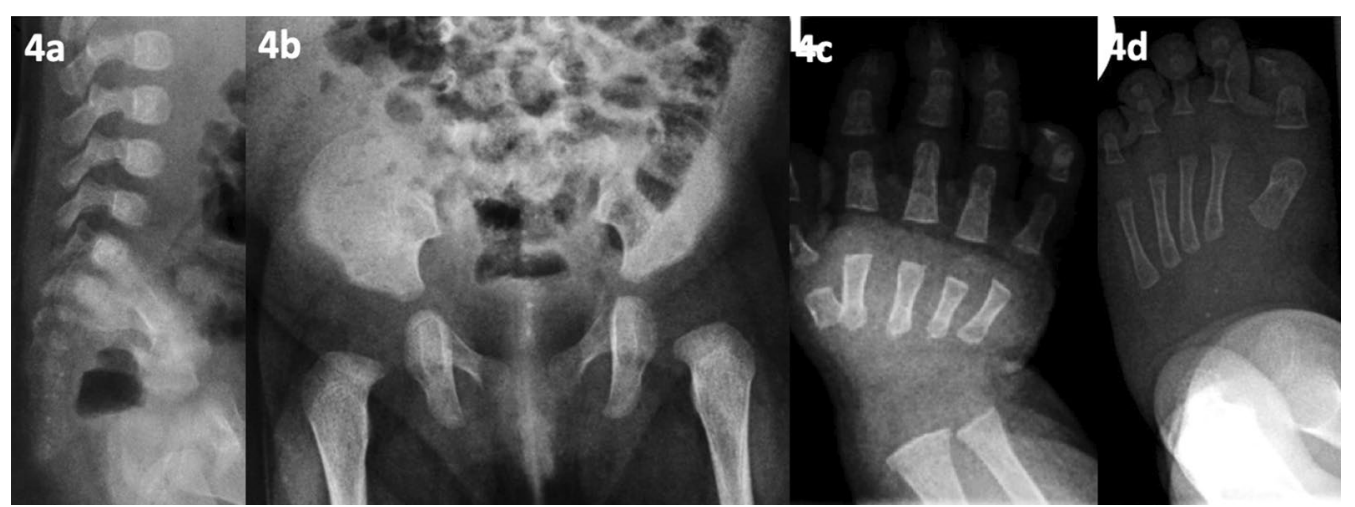

Fig. 4 (patient 8, acquired SD). Aged 2 months; a sacral stippling, small, round/ovoid vertebral bodies with coronal clefts in the thoracic spine. $\mathbf{b}$ Sacral stippling. c short first metacarpals. $\mathbf{d}$ stippled epiphyses distal to the tibia and delayed ossification of the tarsal bones

for CHARGE syndrome (OMIM \#214800) was negative. WES identified two likely pathogenic variants in EXT2 (exostosin glycosyltransferase 2) found in trans with clinical findings overlapping for AREXT2 syndrome (autosomal recessive exostosis-2 gene syndrome). AREXT2 is an ultra-rare recessive disorder with an unclear and expanding phenotype as only four families have been reported
[21]. First described in 2015, it was termed Seizures, Scoliosis and Macrocephaly syndrome (SSM; OMIM \#616682). Later authors suggested the 'AREXT2' label to recognise the lack of uniformity of scoliosis or seizures. It is unclear whether patient 13 has AREXT2 thus, functional work is required. In this unknown syndromal case, WES has ruled out many potential diagnoses and the 


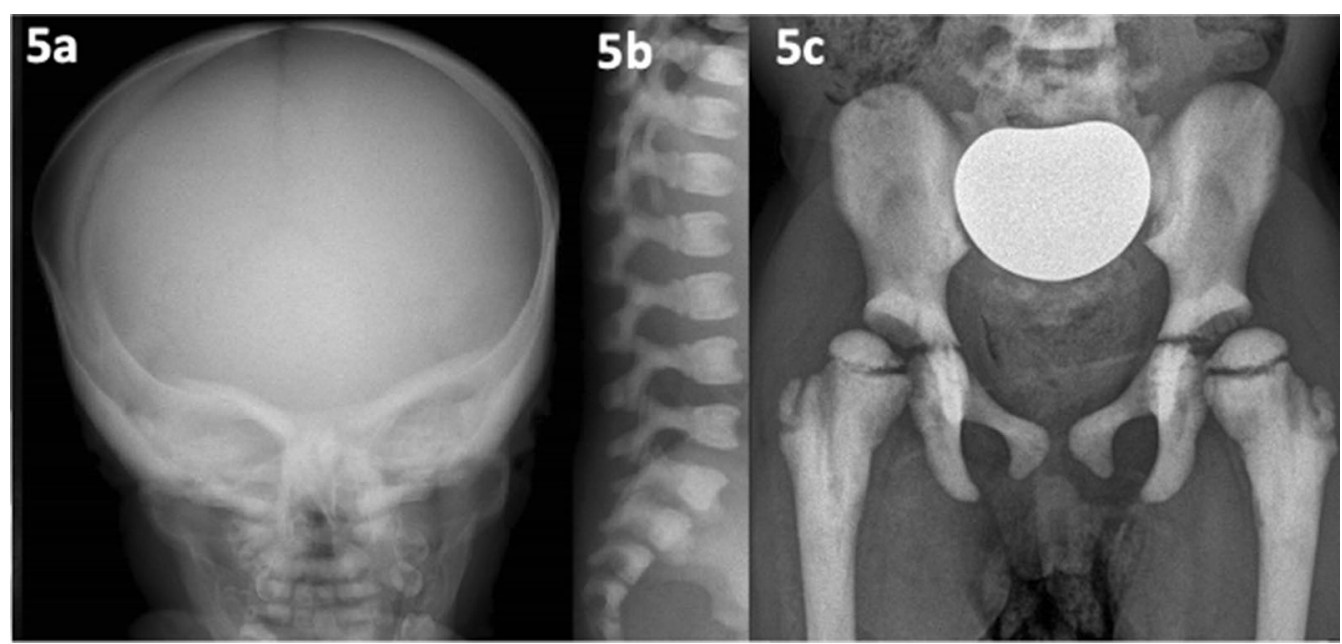

Fig. 5 (patient 9, infantile osteopetrosis). a and b Aged 1 year; thickened cortex skull, bone-in-bone appearance of vertebrae, anterior concavity. $\mathbf{c}$ Age 8 years; sclerotic pelvic bones/femora

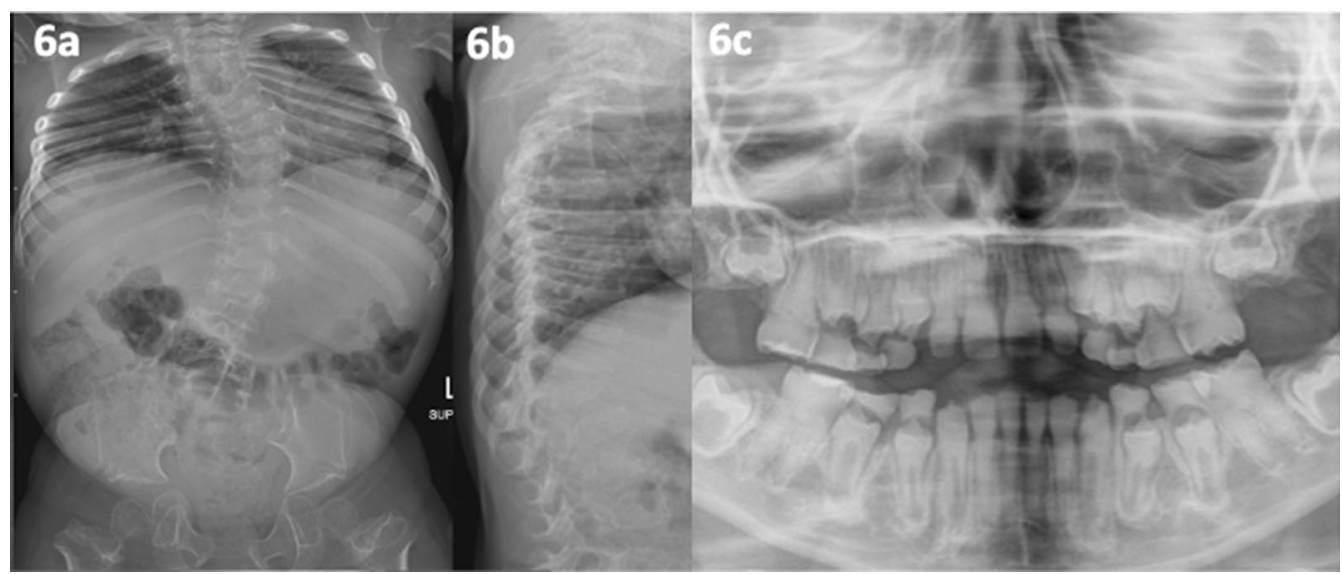

Fig. 6 (patient 11, odontochondrodysplasia). a, b Aged 5.5 years; severe s-shaped scoliosis, marked platyspondyly, segmentation abnormality at T4/5, spondylolisthesis, slender ribs posteriorely with mild anterior rib flaring, proximal femoral metaphyseal dysplasia. c 3 years dentine dysplasia

WES-based agnostic approach allowed for further investigation of an emerging ultra-rare condition.

\section{Theme 5: WES to exclude a genetic diagnosis and support an acquired cause}

Chondrodysplasia punctata (CDP) is a rare SD characterised by punctiform calcification of cartilage and is acquired or genetic in origin [22]. Genetic forms (OMIM \#302950/118650) are heterogeneous. Acquired forms can be due to maternal malabsorption of vitamin $\mathrm{K}$, maternal warfarin or some anticonvulsant [23]. Patient 8, a 2-yearold female, presented with faltering growth and antenatal exposure to lamotrigine and topiramate. Antenatal scans demonstrated short long-bones. Post-natal radiology (see Fig. 4a-d) showed sacral stippling with delayed tarsal ossification, consistent with CDP [24]. Since several genes cause CDP, WES is an appropriate strategy to reasonably exclude underlying genetic causes. Thus, negative WES increases antenatal anticonvulsant exposure as the likely cause. Although there is no reported association of lamotrigine and topiramate, causing stippling, other Hydantoin anticonvulsants (e.g. phenytoin) have been reported to do so [23]. This case highlights the need for early radiography, as stippling is often only seen in the first year of life and rarely after age three [25].

Patient 15 was born with normal birth and growth parameters until age 11 months when an unexplained prolonged fever led to a diagnosis of haemophagocytic 
lymphohistiocytosis (OMIM \#603553). Biallelic PRF1 (Perforin-1) pathogenic variants (compound heterozygote) were confirmed. At 18 months, matched unrelated cord blood HSCT (homologous stem cell transplantation) was performed. Subsequent progressive growth failure developed, associated with radiographic spondyloepimetaphyseal chondrodysplasia (progressive changes as shown in Fig. $7 \mathrm{a}-\mathrm{f}$, note radiographs prior to HSCT were not take as skeletal dysplasia was not suspected then), functional asplenia and sensorineural hearing loss. Development of skeletal changes throughout childhood including fixed flexion hip deformity, marked pes planus, marked genu valgum and pectus carinatum. Extensive endocrine investigations returned no cause. WES (and subsequent WGS) was negative for a skeletal cause. An international group of SD experts noted the similarity of this case and several others, concluding a potentially new disorder manifesting with growth failure and a chondrodysplasia phenocopy post early HSCT for non-oncological disorders [26]. This case highlights the utility of WES to reduce the likelihood of a genetic cause significantly and increase the confidence of an acquired cause [26].

\section{Further discussion}

As many previous studies have discussed, a broad approach to genetic testing through WES or WGS allows for the identification of conditions that may not have been suspected clinically and thus the expansion of previously known phenotypes.

In our cohort of 15 patients, the use of WES has led to three novel findings. Firstly, Patient 7 is the first reported case of MC-D2HGA due to a germline variant. Secondly, patient 13 , is potentially the fifth reported family with AREXT2 syndrome providing further expansion of the phenotype. Lastly, patient 15 , is one of seven patients who have collectively provided evidence for a new disease, 'chondrodysplasia phenocopy post early HSCT for non-oncological disorders' and has been submitted for publication [26].

Although WES remains the current 'go-to' diagnostic test in many rare disease scenarios, we increasingly see a shift to WGS, especially for the acutely ill child. Even then, a large body of undiagnosed patients remain. When WES returns negative or inconclusive results, for many, the diagnostic odyssey is abandoned or halted. Yet it is clear that re-analysis of WES data can often result in diagnosis in $10-15 \%$ of these cases [2].

Additional testing such as long read sequencing (LRS), copy number variants $(\mathrm{CNV})$ in non-coding regions, non-coding variants $(\mathrm{NCV})$, repeat expansion (RE), methylation testing (MT) and other structural changes will need to be explored to increase diagnostic yield. Burdick et al. explored the proportion of diagnoses from additional testing in 54 patients with clinical diagnoses, enrolled in their Undiagnosed Disease Network [2]. Of the 54 participants, the molecular diagnosis was obtained in $36(67 \%)$ through WES, and in $15(28 \%)$ through additional testing. Of these, 7/15 (47\%) had an NCV, 6/15 (40\%) a CNV, and 2/15 (13\%) had a RE or a DNA methylation disorder. A yield figure could not be given since there were many other patients within the programme, who were at different stages of assessment. Nevertheless, the report highlights the benefit of testing beyond WES and the approaches that can be considered.

This combined with the advance of radiomics (the systematic use of artificial intelligence to provide diagnostic processing and analysis of ever sophisticated imaging data) will usher in an increased yield. The careful combination of the physician (endocrinologist, orthopaedic surgeon), radiologist and geneticist working together will be crucial.

The difficulty with large scale sequencings, such as WES or WGS is the generation of large numbers of variants of unknown significance (VUS), especially as genetic testing, is mainstreamed. Likewise, bioinformatic

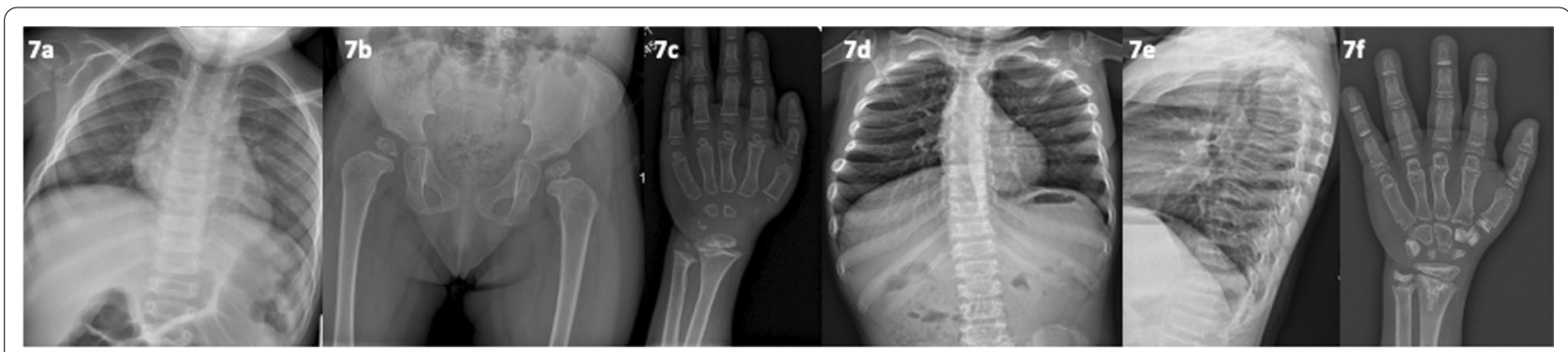

Fig. 7 (patient 15, post HSCT related SD). a-c Aged 5 years; broad ribs, platyspondyly, proximal femoral epiphyseal dysplasia/metaphyseal irregularity. d-f Age 8 years; progressive changes; broadening of ribs, worsening vertebral end-plate irregularities, diffuse platyspondyly, increased density of carpal bones (excluding the hamate and capitate),increase phalangeal epiphyseal density, dense distal ulnar with v-shaped chondroid type calcification extending distally 
pipelines can present de-novo variants with strong computational evidence suggestive of particular conditions, that are not easy to dismiss. The present challenge is, therefore, that of 'variant interpretation'. Further familial segregation is not always possible.

Traditionally, one way to assess a VUS is to perform functional studies (e.g. fresh blood samples to undertake RNA studies to see if the DNA change has a functional impact on RNA production and therefore on the given protein). This method is challenging as it requires fresh blood samples (as RNA degrades quickly), a difficulty, especially in the paediatric population and blood samples are not always the appropriate sample to test a particular variant as the gene of interest may not particularly be expressed.

Additionally, RNA studies are not always possible in the NHS diagnostic lab, and clinicians often had to partner with university academics or other institutions for functional work (often by providing fresh blood samples, or skin samples or saliva). The problems with this are manifold; time-intensive and dependent on knowing suitable partners. Furthermore, obtaining the required sample can be difficult; we have already discussed the difficulty with blood samples. Skin samples require a skin biopsy which is a relatively invasive procedure. Saliva samples are often difficult to work with, of poor quality/limited in what can be assessed. Lastly, with more and more functional study requests to academics, the boundaries of responsibility for what are essentially research analyses are blurred, thus increasing reluctance from academia to perform such work (culpability issue). Suitable new 'quick and easy' tests need developing for variant analysis, and hair pluck analysis may be one such avenue in the SD domain, due to beneficial expression profiles of SD genes.

\section{Conclusion}

Although the genetic causes of $>450$ forms of skeletal disorders have been rapidly uncovered, distinct SD conditions of unknown genetic aetiology remain [27]. The best strategy for identifying these may be the unbiased approach of WES or WGS, especially in children and patients with severe or multi-systemic diseases. It can be particularly beneficial to detect de novo pathogenic variants using a trio design. In sporadic cases, analysis of trios may reveal de-novo pathogenic variants. In familial cases, a combination of WES and either WGS or SNP typing could yield linkage information for prioritisation of rare variants [28]. If no variants are identified through WES, then further testing is needed, though the costeffectiveness of such studies needs determination [2].

Our results highlight the cost-effective use of WEStargeted bioinformatic analysis as a diagnostic tool for $\mathrm{SD}$, particularly for patients with presumed SD, where detailed phenotyping is essential. The thorough clinical evaluative approach and planning between clinical, radiological, and molecular teams is essential for improved service provision.

As we move towards WGS trio sequencing, experience and this study shows that careful categorisation of patients of those with known pre-test diagnosis will yield a higher return of molecular confirmation $(8 / 10$ in this study) as opposed to unknown diagnosis pre-test $(1 / 5$ in this study) thus highlighting the importance of carefully selecting the most suitable cases for highest WGS yield. With the evolution of this pilot study, the SD panel was extended to 498 genes and anecdotally several more pretest unknown SD cases were molecularly resolved, thus it is felt WES/WGS will play a more significant role in cases with no prior test diagnosis.

The clinical yield of WES will increase over time, allowing providers to arrive at a diagnosis efficiently. This information will help avoid needless diagnostic procedures/costly additional tests and individualised patient care, allowing for informative clinical decisions and reassurance to patients and families [13]. More diagnoses will, in turn, lead to more information on natural history, improved mechanistic details, and hopefully increased therapies and clinical trials.

\section{Appendix 1: The skeletal dysplasia gene panel v1 (222 genes, 'Pan 272') in accordance with UK Genetic Testing Network}

ACAN, ACP5, ACVR1, ADAMTSL2, AGPS, ALPL, ALX1, ALX3, ALX4, ANKH, ANO5, ANTXR2, ARHGAP31, ARSE, ATP6V0A2, B3GALT6, B4GALT7, BMP1, BMP2, BMPR1B, CA2, CANT1, CASR, CC2D2A, CCDC8, CDC6, CDH3, CDKN1C, CDT1, CEP290, CHST14, CHST3, CHSY1, CLCN5, CLCN7, COL10A1, COL11A1, COL11A2, COL1A1, COL1A2, COL2A1, COL9A1, COL9A2, COL9A3, COMP, CREB3L1, CRTAP, CTSK, CUL7, DDR2, DHCR24, DLL3, DLX3, DMP1, DYM, DYNC2H1, EBP, EFNB1, EFTUD2, EIF2AK3, ENPP1, ESCO2, EVC, EVC2, EXT1, EXT2, FAM20C, FBLN1, FBN1, FBXW4, FERMT3, FGF10, FGF23, FGF9, FGFR1, FGFR2, FGFR3, FKBP10, FLNA, FLNB, FMN1, FOXC1, GALNT3, GDF5, GLI3, GNAS, GORAB, GPC6, GPX4, GREM1, HDAC4, HOXA11, HOXD13, HPGD, HSPG2, ICK, IFITM5, IFT122, IFT140, IFT172, IFT43, IFT80, IFT88, IHH, IKBKG, IL1RN, INPPL1, KAT6B, KIF22, KIF7, LBR, LEMD3, LEPRE1, LFNG, LIFR, LMBR1, LMNA, LMX1B, LRP4, LRP5, MAFB, MATN3, MESP2, MGP, MKS1, MMP13, MMP2, MMP9, MSX2, MYCN, NEK1, NIPBL, NKX3-2, NLRP3, NOG, NOTCH2, NPR2, NSDHL, OBSL1, OFD1, ORC1, ORC4, ORC6, 
OSTM1, PAPSS2, PCNT, PEX7, PHEX, PIGV, PITX1, PLEKHM1, PLOD2, POLR1C, POR, PPIB, PRKAR1A, PTDSS1, PTH1R, PTHLH, PTPN11, PYCR1, RAB23, RASGRP2, RECQL4, ROR2, RPGRIP1L, RUNX2, SALL1, SALL4, SBDS, SERPINF1, SERPINH1, SH3BP2, SH3PXD2B, SHH, SHOX, SLC25A12, SLC26A2, SLC34A3, SLC35D1, SLC39A13, SMARCAL1, SOST, SOX9, SP7, SULF1, TBCE, TBX15, TBX3, TBX4, TBX5, TBXAS1, TCIRG1, TCOF1, TCTN3, TGFB1, THPO, TMEM216, TMEM38B, TMEM67, TNFRSF11A, TNFRSF11B, TNFSF11, TP63, TRAPPC2, TREM2, TRIP11, TRPS1, TRPV4, TTC21B, TWIST1, TWIST2, TYROBP, WDR19, WDR34, WDR35, WDR60, WISP3, WNT3, WNT5A, WNT7A, ZMPSTE24.

\section{Abbreviations}

aCGH: Array comparative genomic hybridisation; ACH: Achondroplasia; AMD: Acromesomelic dysplasia; AR: Autosomal recessive; AREXT2: Autosomal recessive exostosin glycosyltransferase 2; CCHD: Complex congenital heart disease; CD: Campomelic dysplasia; CDP: Chondrodysplasia punctata; CHD7: Chromodomain helicase DNA binding protein 7; CLCN7: Chloride voltagegated channel 7; COL11A2: Collagen type XI alpha-2 chain; COL2A1: Collagen type II alpha-1 chain; COL9A1-COL9A3: Collagen type IX alpha 1-3 chain; COMP: Cartilage oligomeric matrix protein; DD: Developmental delay; EC: Enchondromatosis; EDS: Ehlers-Danlos syndrome; EVS: Exome variant server; EXT2: Exostosin glycosyltransferase 2; FBCG2: Fibrochondrogenesis type 2; GH: Growth hormone; HPO: Human phenotype ontology; HSCT: Homologous stem cell transplantation; IDH1: Isocitrate dehydrogenase 1; IUGR: Intrauterine growth restriction; MATN3: Matrilin-3; MC-D2HGA: Metaphyseal chondromatosis with D-2-hydroxyglutaric aciduria; MED: Multiple epiphyseal dysplasia; MPOD: Microcephalic osteodysplastic primordial dwarfism; OSMED: Otospondylomegaepiphyseal dysplasia; PAGE: Prenatal assessment of genomes and exomes; PEG: Percutaneous endoscopic gastrostomy; PCNT: Pericentrin; PRF1: Perforin-1; PP1: Protein phosphate-1; PP4: Protein phosphate-4; SD: Skeletal dysplasia; SEDC: Spondyloepiphyseal dysplasia congenita; SEDT: Spondyloepiphyseal dysplasia tarda; SOX-9: SRY (sex determining region Y)-Box 9; SSM: Seizures, scoliosis and macrocephaly syndrome; TES: Targeted exome sequencing; TRAPPC2: Tracking protein particle complex, subunit 2; TRIP11: Thyroid receptor-interacting protein 11; VUS: Variant of unknown significance; WES: Whole-exome sequencing; WGS: Whole-genome sequencing; 100kGP: 100,000 Genomes project.

\section{Acknowledgements}

With thanks to patients and families.

\section{Authors' contributions}

Manuscript prepared, conceptualised and researched by AS, JS and EM helped prepare the manuscript, $\mathrm{AC} / \mathrm{AO} / \mathrm{CH}$ reviewed radiological imaging, $\mathrm{MC} / \mathrm{AC} /$ MI/ABM provided clinical care and phenotyping, MJ/MY/YP/SL provided laboratory support/variant analysis, Ml devised the project, obtained grant funding and supervised the manuscript. All authors read and approved the final manuscript.

\section{Funding}

Grant support of $£ 15,000$ in 2013 from the British Skeletal Dysplasia Group Project for whole-exome sequencing of 15 patients.

\section{Availability of data and materials}

The datasets generated during and/or analysed during the current study are not publicly available due to maintaining patient confidentiality but are available from the corresponding author on reasonable request.

\section{Declarations}

\section{Ethics approval and consent to participate}

Ethics committee review for our cohort was obtained. Approval number 08/ H0810/14-National Bromley Research Ethics Committee at Guy's and St. Thomas's Hospitals NHS Foundation Trust. Clinical/personal patient data was obtained as part of clinical care. Informed consent (written) to participate was obtained from the parents/legal guardians of all participants in the study. [This is a clinical paper not a research study hence an IRB was not involved].

\section{Consent for publication}

Patient privacy and confidentiality have been protected. All patients were study participants at the Guy's and St Thomas' Hospital clinical site. Authors have full written consent to publish the information from the patient(s) or their parent(s)/legal guardian(s) prior to submission.

\section{Competing interests \\ None}

\section{Author details}

'Department of Clinical Genetics, Guy's and St Thomas' NHS Foundation Trust, London, UK. ${ }^{2}$ College of Medical and Dental Sciences, University of Birmingham, Birmingham, UK. ${ }^{3}$ The Royal Wolverhampton NHS Trust, Wolverhampton, UK. ${ }^{4}$ Radiology Department, Great Ormond Street Hospital for Children, NHS Foundation Trust, London, UK. ${ }^{5}$ Clinical Genetics Department, University Hospitals Bristol and Weston, Bristol, UK. ${ }^{6}$ Department of Paediatric Endocrinology, Evelina London Children's Hospital, London, UK. Viapath LLP, Guy's Hospital, 5th Floor Tower Wing, London SE1 9RT, UK. ${ }^{8}$ Neurogenetics, Rare and Inherited Disease Laboratory, North Thames GLH, Barclay House, 37 Queen Square, London WC1N 3BH, UK. ${ }^{9}$ Great Ormond Street Hospital for Children, London, UK. ${ }^{10}$ Emeritus Professor of Paediatric Radiology, Institute of Child Health, University of London, London, UK. ${ }^{11}$ Academic Unit of Child Health, University of Sheffield, Sheffield, UK. ${ }^{12}$ Division of Genetics and Molecular Medicine, King's College London School of Medicine, London, UK.

Received: 2 December 2020 Accepted: 28 May 2021

Published online: 06 June 2021

\section{References}

1. Amberger JS, Bocchini CA, Schiettecatte F, Scott AF, Hamosh A. OMIM.org: Online Mendelian Inheritance in Man $\left(\mathrm{OMIM}^{\circledR}\right)$, an Online catalog of human genes and genetic disorders. Nucleic Acids Res. 2015;43(D1):D789-98.

2. Burdick KJ, Cogan JD, Rives LC, Robertson AK, Koziura ME, Brokamp E, et al. Limitations of exome sequencing in detecting rare and undiagnosed diseases. Am J Med Genet Part A. 2020;182(6):1400-6. https://doi. org/10.1002/ajmg.a.61558.

3. Liu HY, Zhou L, Zheng MY, Huang J, Wan S, Zhu A, et al. Diagnostic and clinical utility of whole genome sequencing in a cohort of undiagnosed Chinese families with rare diseases. Sci Rep 2019;9(1).

4. Yang Y, Muzny DM, Reid JG, Bainbridge MN, Willis A, Ward PA, et al. Clinical whole-exome sequencing for the diagnosis of mendelian disorders. $\mathrm{N}$ Engl J Med. 2013;369(16):1502-11.

5. Beaulieu CL, Majewski J, Schwartzentruber J, Samuels ME, Fernandez BA, Bernier FP, et al. FORGE Canada consortium: outcomes of a 2-year national rare-disease gene-discovery project. Am J Hum Genet. 2014;94(6):809-17.

6. Taylor JC, Martin HC, Lise S, Broxholme J, Cazier JB, Rimmer A, et al. Factors influencing success of clinical genome sequencing across a broad spectrum of disorders. Nat Genet. 2015;47(7):717-26.

7. Tan TY, Dillon OJ, Stark Z, Schofield D, Alam K, Shrestha R, et al. Diagnostic impact and cost-effectiveness of whole-exome sequencing for ambulant children with suspected monogenic conditions. JAMA Pediatr. 2017;171(9):855-62

8. Meng L, Pammi M, Saronwala A, Magoulas P, Ghazi AR, Vetrini F, et al. Use of exome sequencing for infants in intensive care units ascertainment of severe single-gene disorders and effect on medical management. JAMA Pediatr 2017;171(12). 
9. Farnaes L, Hildreth A, Sweeney NM, Clark MM, Chowdhury S, Nahas S, et al. Rapid whole-genome sequencing decreases infant morbidity and cost of hospitalization. npj Genomic Med 2018;3(1).

10. Wright CF, FitzPatrick DR, Firth HV. Paediatric genomics: diagnosing rare disease in children. Nat Rev Genet. 2018;19(5):253-68.

11. Wright CF, McRae JF, Clayton S, Gallone G, Aitken S, FitzGerald TW, et al. Making new genetic diagnoses with old data: iterative reanalysis and reporting from genome-wide data in 1,133 families with developmental disorders. Genet Med. 2018;20(10):1216-23.

12. Zanelli S. Skeletal dysplasia. eMedicine J. 2018.

13. Retterer K, Juusola J, Cho MT, Vitazka P, Millan F, Gibellini F, et al. Clinical application of whole-exome sequencing across clinical indications. Genet Med. 2016:18(7):696-704.

14. Bae JS, Kim NKD, Lee C, Kim SC, Lee HR, Song HR, et al. Comprehensive genetic exploration of skeletal dysplasia using targeted exome sequencing. Genet Med. 2016;18(6):563-9.

15. Lord J, McMullan DJ, Eberhardt RY, Rinck G, Hamilton SJ, QuinlanJones $\mathrm{E}$, et al. Prenatal exome sequencing analysis in fetal structural anomalies detected by ultrasonography (PAGE): a cohort study. Lancet. 2019:393(10173):747-57.

16. UK Genetic Testing Network. National Genomic Test Directory: testing criteria for rare and inherited disease. 2019.

17. Bonafé $L$, Mittaz-Crettol L, Ballhausen D, Superti-Furga A. Multiple epiphyseal dysplasia, recessive. GeneReviews ${ }^{\circledR}$. University of Washington, Seattle; 1993.

18. Briggs MD, Wright MJ, Mortier GR. Multiple epiphyseal dysplasia, autosomal dominant. GeneReviews ${ }^{\circledR}$. University of Washington, Seattle; 1993.

19. Pansuriya TC, Kroon HM, Bovée JVMG. Enchondromatosis: insights on the different subtypes. Int J Clin Exp Pathol. 2010;3(6):557-69.

20. Srinivasan A, Zhou Y, Scordino T, Prabhu S, Wierenga A, Simon G, et al. IDH1 mutated acute myeloid leukemia in a child with metaphyseal chondromatosis with D-2-hydroxyglutaric aciduria. Pediatr Hematol Oncol. 2020;37(5):431-7.
21. Gupta A, Ewing SA, Renaud DL, Hasadsri L, Raymond KM, Klee EW, et al. Developmental delay, coarse facial features, and epilepsy in a patient with EXT2 gene variants. Clin Case Rep. 2019;7(4):632-7.

22. Irving MD, Chitty LS, Mansour S, Hall CM. Chondrodysplasia punctata: a clinical diagnostic and radiological review. Clin Dysmorphol. 2008;17(4):229-41.

23. Wessels MW, Den Hollander NJ, De Krijger RR, Nikkels PGJ, Brandenburg $H$, Hennekam R, et al. Fetus with an unusual form of nonrhizomelic chondrodysplasia punctata: case report and review. Am J Med Genet. 2003; 120A(1):97-104

24. Castriota-Scanderbeg A, Dallapiccola B. Abnormal skeletal phenotypes: from simple signs to complex diagnoses. Abnormal skeletal phenotypes: from simple signs to complex diagnoses. Springer Berlin Heidelberg; 2005; 1-962 p.

25. Firth H V., Hurst JA. Clinical genetics and genomics (Oxford Desk Reference). Oxford University Press; 2017.

26. Botto L, Meeths M, Campos-Xavier B, Bergamaschi R, Mazzanti L, Scarano E, et al. Chondrodysplasia and growth failure in children following early hematopoietic stem cell transplantation for non-oncologic disorders. Am J Med Genet A. 2021;185(2):517-27.

27. Mortier GR, Cohn DH, Cormier-Daire V, Hall C, Krakow D, Mundlos S, et al. Nosology and classification of genetic skeletal disorders: 2019 revision. Am J Med Genet Part A. 2019;179(12):2393-419.

28. Geister KA, Camper SA. Advances in skeletal dysplasia genetics. Annu Rev Genom Hum Genet. 2015;16:199-227.

\section{Publisher's Note}

Springer Nature remains neutral with regard to jurisdictional claims in published maps and institutional affiliations.
Ready to submit your research? Choose BMC and benefit from:

- fast, convenient online submission

- thorough peer review by experienced researchers in your field

- rapid publication on acceptance

- support for research data, including large and complex data types

- gold Open Access which fosters wider collaboration and increased citations

- maximum visibility for your research: over $100 \mathrm{M}$ website views per year

At BMC, research is always in progress.

Learn more biomedcentral.com/submissions 